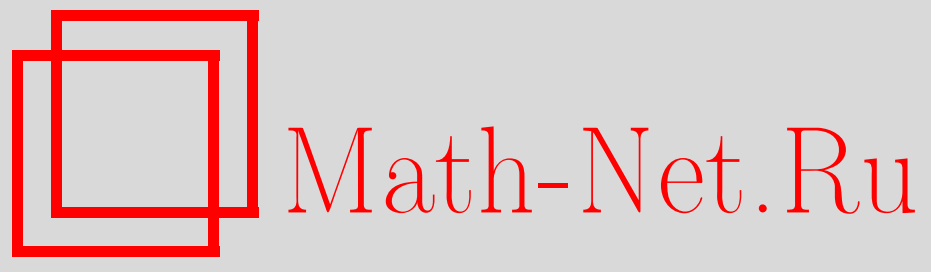

Е. А. Горский, Симметрии мотивных интегралов, УМH, 2008, том 63, выпуск 4, 179-180

DOI: https://doi.org/10.4213/rm9223

Использование Общероссийского математического портала Math-Net.Ru подразумевает, что вы прочитали и согласны с пользовательским соглашением http://www . mathnet.ru/rus/agreement

Параметры загрузки:

IP: 54.209 .52 .79

26 апреля 2023 г., 14:28:11

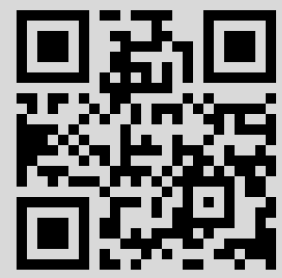




\section{Симметрии мотивных интегралов}

\section{Е. А. Горский}

Пусть $\mu(\gamma)$ - число Милнора [1] ростка плоской кривой $\gamma(t)=(x(t), y(t))$. Рассмотрим семейство мотивных интегралов [2] $G_{k, m}(t)=\int_{\{\operatorname{Ord} x(t)=k, \operatorname{Ord} y(t)=m\}} t^{\mu(\gamma)} d \gamma$ по пространству параметризованных дуг на плоскости $\left(\mathbb{C}^{2}, 0\right)$.

УтВеРждения. 1. $G_{k, m}(t)$ - рационалъная функция от $t$ и класса афбинной прямой $\mathbb{L}$.

2. $G_{1,1}(t)=(\mathbb{L}-1)^{2} \mathbb{L}^{-2}$.

3. $G_{k, m}(t)=G_{m, k}(t)$.

4. $G_{k, m}(t)=t^{k(k-1)} \mathbb{L}^{-k} G_{k, m-k}(t)$ npu $m>k$.

5. $G_{k, k}(t)=(\mathbb{L}-1) \sum_{m>k} G_{k, m}(t)$.

6. $G_{k, k}(t)=\frac{(\mathbb{L}-1) t^{k(k-1)} \mathbb{L}^{-k}}{1-t^{k(k-1)} \mathbb{L}^{1-k}} \sum_{m<k} G_{k, m}(t)$.

ДоказАтельство. Утверждения 2 и 3 очевидны. Утверждение 4 следует из формулы замены переменных в мотивном интеграле ([2], см. также [3]). Утверждение 5 следует из того факта, что если порядки $x(t)$ и $y(t)$ равны, то $y(t)=\lambda x(t)+y^{\prime}(t)$, где $\lambda$ - ненулевое число, а порядок $y^{\prime}(t)$ больше порядка $y(t)$. Докажем утверждение 6 :

$$
\begin{aligned}
G_{k, k}(t) & =(\mathbb{L}-1) \sum_{m>k} G_{k, m}(t)=(\mathbb{L}-1)^{2} \sum_{m>k} t^{k(k-1)} \mathbb{L}^{-k} G_{k, m-k} \\
& =(\mathbb{L}-1)^{2} \sum_{m>k} t^{k(k-1)} \mathbb{L}^{-k}\left(\sum_{m<k} G_{k, m}(t)+G_{k, k}(t)+\sum_{m>k} G_{k, m}(t)\right) \\
& =(\mathbb{L}-1)^{2} \sum_{m>k} t^{k(k-1)} \mathbb{L}^{-k}\left(\sum_{m<k} G_{k, m}(t)+G_{k, k}(t)\left(1+\frac{1}{\mathbb{L}-1}\right)\right),
\end{aligned}
$$

откуда и следует искомый результат. Утверждение 1 следует из утверждений 2-4 и 6 .

ОпредЕлЕниЕ. Введем следующие многочлены от двух переменных:

$$
S_{a, k}\left(t, \mathbb{L}^{-1}\right)=\sum_{1 \leqslant m<k, \text { НОД }(m, k)=a} t^{(k-1)(m-1)-(a-1)^{2}} \mathbb{L}^{2 a-k-m} .
$$

УтвеРЖДЕНИЯ. 7. Если НОД $(k, m)=a, m o$

$$
G_{k, m}(t, \mathbb{L})=t^{(k-1)(m-1)-(a-1)^{2}} \mathbb{L}^{2 a-k-m} G_{a, a}(t, \mathbb{L}) .
$$

8. Имеет место равенство

$$
G_{k, k}(t, \mathbb{L})=\frac{(\mathbb{L}-1) t^{k(k-1)} \mathbb{L}^{-k}}{1-t^{k(k-1)} \mathbb{L}^{1-k}} \sum_{a \mid k} S_{a, k}\left(t, \mathbb{L}^{-1}\right) G_{a, a}(t) .
$$

9. Имеет место равенство

$$
G_{k, k}(t, \mathbb{L})=\sum_{1=a_{0}, a_{1}, \ldots, a_{r}=k} \frac{(\mathbb{L}-1)^{2+r} t^{\sum_{j=1}^{r} a_{j}\left(a_{j}-1\right)} \mathbb{L}^{-2-\sum_{j=1}^{r} a_{j}}}{\prod_{j=1}^{r}\left(1-t^{a_{j}\left(a_{j}-1\right)} \mathbb{L}^{1-a_{j}}\right)} \prod_{j=1}^{r} S_{a_{j-1}, a_{j}}\left(t, \mathbb{L}^{-1}\right),
$$

где суммирование ведется по всем наборам $\left(1=a_{0}<a_{1}<\cdots<a_{r}=k\right)$, в которых $a_{j-1} \mid a_{j}$ для всех $j$.

Работа выполнена при финансовой поддержке грантов РФФИ-007-00593, INTAS-05-7805, НШ-4719.2006.1, а также Фонда поддержки молодых ученых "Конкурс Мёбиуса". 
ДокАЗАтельство. Заметим, что $(k-1)(m-1)=k(k-1)+k(m-k-1)$, поэтому, так как алгоритм Евклида для $k$ и $m$ сходится к $a$, то из свойства 4 получаем утверждение 7. Подставляя (1) в утверждение 6 , получаем утверждение 8 . Применяя утверждение 7 до тех пор, пока не дойдем до $a=1$, и используя утверждение 2 , получим утверждение 9.

Из формулы обращения Мёбиуса вытекает следующее

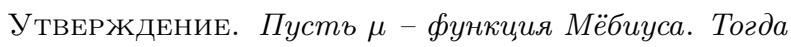

$$
S_{a, k}\left(t, \mathbb{L}^{-1}\right)=t^{-(a-1)^{2}} \mathbb{L}^{2 a} \sum_{a|b, b| k} \mu\left(\frac{b}{a}\right) \frac{t^{(k-1)(b-1)} \mathbb{L}^{-k-b}-t^{(k-1)^{2}} \mathbb{L}^{-2 k}}{1-t^{(k-1) b} \mathbb{L}^{-b}} .
$$

Подставим (2) в утверждение 9 и приведем подобные члены. Получим следующий результат.

Теорема 1. Пусть $a=$ НОД $(k, m)$. Тогда

$$
\begin{aligned}
G_{k, m}(t, \mathbb{L})= & \int_{\{\operatorname{Ord} x(t)=k, \operatorname{Ord} y(t)=m\}} t^{\mu(\gamma)} d \gamma=(\mathbb{L}-1)^{2} t^{(k-1)(m-1)} \mathbb{L}^{-k-m} \\
& \times \sum_{\underline{a}, \underline{b}} \prod_{j=1}^{r} \mu\left(\frac{b_{j}}{a_{j-1}}\right) \frac{(\mathbb{L}-1) t^{\left(a_{j}-1\right) b_{j}} \mathbb{L}^{-b_{j}}\left(1-t^{\left(a_{j}-1\right)\left(a_{j}-b_{j}\right)} \mathbb{L}^{b_{j}-a_{j}}\right)}{\left(1-t^{a_{j}\left(a_{j}-1\right)} \mathbb{L}^{1-a_{j}}\right)\left(1-t^{\left(a_{j}-1\right) b_{j}} \mathbb{L}^{-b_{j}}\right)},
\end{aligned}
$$

где суммирование ведется по всем наборам $\left(1=a_{0} \leqslant b_{1}<a_{1} \leqslant \cdots<a_{r-1} \leqslant b_{r}<\right.$ $a_{r}=a$ ), в которых $a_{j-1}\left|b_{j} u b_{j}\right| a_{j}$ для всех $j$ (число $r$ не фиксировано).

Напомним, что в левой части (3) $\mu$ обозначает число Милнора, а в правой - функцию Мёбиуса, что, впрочем, не должно приводить к недоразумениям.

СледСтвиЕ 1. $G_{k, m}\left(t^{-1}, \mathbb{L}^{-1}\right)=t^{-2(k-1)(m-1)} \mathbb{L}^{2 k+2 m-2} G_{k, m}(t, \mathbb{L})$.

ДокАЗАтЕльство. Это утверждение следует из того, что каждый из $r$ сомножителей в правой части (3) инвариантен относительно замены $(t, \mathbb{L}) \mapsto\left(t^{-1}, \mathbb{L}^{-1}\right)$.

\section{Список литературы}

[1] В. И. Арнольд, А.Н. Варченко, С. М. Гусейн-Заде, Особенности дифференцируемъх отображений, Наука, M., 1982; англ. пер.: V. I. Arnold, S. M. Gusejn-Zade, A. N. Varchenko, Singularities of differentiable maps. Vol. I. The classification of critical points, caustics and wave fronts, Monogr. Math., 82, Birkhäuser, Boston, 1985; Vol. II. Monodromy and asymptotics of integrals, Monogr. Math., 83, Birkhäuser, Boston, 1988. [2] J. Denef, F. Loeser, Invent. Math., 135:1 (1999), 201-232. [3] Е. А. Горский, Алгебра и анализ, 19:4 (2007), 92-111; англ. пер.: E. A. Gorskii, St. Petersburg Math. J., 19 (2008), 561-575.

\section{Е. А. Горский (Е. А. Gorskii)}

Московский государственный университет им. М. В. Ломоносова

E-mail: gorsky@mccme.ru
Представлено С. М. Гусейн-Заде Принято редколлегией 20.06.2008 